\title{
Comparative pharmacokinetic and pharmacodynamic evaluation of branded and generic formulations of meloxicam in healthy male volunteers
}

\author{
This article was published in the following Dove Press journal: \\ Therapeutics and Clinical Risk Management \\ 23 July 2013 \\ Number of times this article has been viewed
}

\author{
Mario Del Tacca ${ }^{1,2}$ \\ Giuseppe Pasqualetti ${ }^{3}$ \\ Giovanni Gori' \\ Pasquale Pepe' \\ Antonello Di Paolo² \\ Marianna Lastella ${ }^{2}$ \\ Ferdinando De Negri' \\ Corrado Blandizzi \\ 'Clinical Pharmacology Centre for \\ Drug Experimentation, Pisa University \\ Hospital, ${ }^{2}$ Department of Clinical and \\ Experimental Medicine, ${ }^{3}$ Geriatrics \\ Unit, University of Pisa, Pisa, Italy
}

\begin{abstract}
Purpose: The primary aim of the present study was to assess the pharmacokinetic bioequivalence between a generic formulation of meloxicam $15 \mathrm{mg}$ tablets (Meloxicam Hexal) and its respective brand product (Mobic), in order to verify whether the generic product conforms to the regulatory standards of bioequivalence in the postmarketing setting. As a secondary exploratory aim, the pharmacodynamic effects of the two formulations were also evaluated by means of rating scales following hyperalgesia induced by cutaneous freeze injury.
\end{abstract}

Subjects and methods: A single $15 \mathrm{mg}$ dose of generic or branded meloxicam tablets was administered to 24 healthy male volunteers in a crossover fashion. Plasma samples, collected for 24 hours after dosing, were assayed for meloxicam concentration by a validated highperformance liquid chromatography method.

Results: The analysis of pharmacokinetic parameters did not show any significant difference between the two meloxicam formulations: the $90 \%$ confidence intervals fell within the acceptance range of $80 \%-125 \%(0.84-1.16$ for area under the curve [0-24], and $0.89-1.23$ for peak concentration). No difference in the pharmacodynamic end point was observed between the two groups.

Conclusion: The pharmacokinetic profiles of the two meloxicam formulations confirm the regulatory criteria for bioequivalence; pharmacodynamic data indicate a similar antihyperalgesic effect. The two formulations can be used interchangeably in the clinical setting.

Keywords: meloxicam, pharmacokinetics, healthy volunteers, generic drug, bioequivalence, postmarketing

\section{Introduction}

The purpose of assessing bioequivalence is to demonstrate equivalence in biopharmaceutic quality between the generic medicinal product and the reference branded formulation, in order to allow bridging of preclinical tests and clinical trials associated with the patented original product. Accordingly, pharmaceutical companies, involved in the development of generic drugs, are allowed to follow abbreviated procedures to demonstrate bioequivalence between brand and generic formulations, without the need of repeating a complete, and expensive program of pharmacological and clinical development.

In the Italian setting, open access to preregistration documentation of generic drugs is not allowed, and, on the basis of European Medicines Agency (EMA) guidance, an immediate-release oral formulation of a generic copy (including nonsteroidal
Correspondence: Mario Del Tacca Clinical Pharmacology Centre for Drug Experimentation, Pisa University Hospital, 67 Via Roma, Pisa 56100, Italy

Tel +39050993954

Fax +39050993 431

Email m.deltacca@gmail.com 
anti-inflammatory drugs) might be approved as a biowaiver, by means of in vitro dissolution tests, without performing any clinical investigation. ${ }^{1}$ It is also being increasingly acknowledged that the regulatory criteria for assuming bioequivalence between branded and generic drugs might be insufficient at times, and this is an important issue deserving consideration, in order to ensure full therapeutic equivalence in the postmarketing period. Therefore, performing postmarketing investigations on generic drugs appears to be important to control the quality of marketed formulations. Of note, some postmarketing studies are raising doubts on the actual therapeutic interchangeability between branded and generic drugs available in the pharmaceutical market. For instance, in a postmarketing pharmacokinetic (PK) crossover trial on two generic amoxicillin formulations, a lack of interchangeability was found for one generic product. ${ }^{2}$ Moreover, some authors ${ }^{3-5}$ argued that switching from brand antiepileptics to generic copies might result in an increased risk of therapeutic failure (breakthrough seizures) or adverse reactions. Other examples of treatment failure, with marketed antibiotic, ${ }^{6}$ anticoagulant, ${ }^{7}$ proton-pump inhibitor, ${ }^{8,9}$ antiarrhythmic, ${ }^{10}$ or antidepressant ${ }^{11}$ generic products, have also been published. Thus, the validity of current criteria for assessing interchangeability of generic and branded drugs is being questioned, at least in some instances, and it is becoming evident that inadequate programs for postmarketing quality control of medicinal products might increase the risk of therapeutic failures and/or safety concerns in patients. ${ }^{12}$ Moreover, generic drug use is steadily increasing in Italy, particularly in Tuscany, where the regional health authority strongly supports the prescription of such products in order to reduce health-care costs.

Based on the above considerations, our Clinical Pharmacology Centre for Drug Experimentation has implemented a research program to perform clinical studies aimed at verifying the quality of generic drugs available in the Italian pharmaceutical market, owing to specific scientific interest on the quality of generic drugs prescribed in current clinical practice.

Since nonsteroidal anti-inflammatory drugs represent one of the most prescribed pharmaceutical classes in Italy, we selected Mobic (Boehringer Ingelheim $\mathrm{GmbH}$, Ingelheim, Germany) as a branded meloxicam formulation and randomly selected Meloxicam Hexal among the generic compounds, both marketed in Italy, in order to evaluate whether the generic formulation fulfills the criteria for clinical PK bioequivalence versus its reference branded product.
The choice to indicate both the originator (Mobic) and generic (Meloxicam Hexal) meloxicam brand names was due to transparency reasons and the lack of any conflict of interest between the respective drug companies and the authors. Moreover, we deemed it interesting to perform an exploratory pharmacodynamic (PD) study on the two meloxicam formulations, in order to assess their analgesic effects and compare their $\mathrm{PD} / \mathrm{PK}$ relationship. In this regard, the PD test was carried out by estimating the mechanical pain threshold of cutaneous freeze injury with a von Frey device. ${ }^{13}$

\section{Materials and methods Subjects}

Twenty-four healthy male volunteers were recruited at the Clinical Pharmacology Centre for Drug Experimentation of the University Hospital of Pisa (Italy). The exclusion of women from participating in the present study was due to sexdependent differences in the PK of meloxicam, as previously reported by Meineke and Türck, ${ }^{14}$ who demonstrated that both age and sex significantly affected meloxicam clearance.

In addition, this study investigated the relationship between the PK and PD of meloxicam in healthy volunteers, and therefore the exclusion of females was justified by the known hormonal influence on pain tolerance and threshold in menstruating women. ${ }^{15-18}$ At the same time, the exclusion of women might be regarded as a limitation, since a more heterogeneous study population might have increased the generalization of our findings. Eligible subjects were aged 18-50 years, had a body mass index (BMI) between 19 and $25 \mathrm{~kg} / \mathrm{m}^{2}$, did not smoke, and had an unremarkable clinical history. Subjects with history or evidence of renal, gastrointestinal, hepatic, or hematologic abnormalities, any acute or chronic disease, or any drug allergy were excluded. Clinical evaluations and tests included: medical history, physical examination, height, weight, BMI, vital signs (heart rate, systolic and diastolic blood pressure, and body temperature), electrocardiogram, renal and liver-function tests, urinalysis, and urine drug and alcohol testing. Volunteers were requested to report any abnormality occurring throughout and after the study. Subjects were required to be negative for human immunodeficiency virus (HIV) and hepatitis B and C viruses.

At the time of enrollment, the volunteers were informed of the purpose, duration, and risks of the study, and they were requested to sign a written informed consent. They were not allowed to consume alcohol, or beverages and foods containing caffeine from 48 hours prior to drug administration until the end of the study. All participants were requested to fast for 10 hours before drug dosing, in 
order to minimize the PK variability and allow appropriate comparisons with data available in the literature. ${ }^{19-22}$ The dietary regimen was the same for all subjects in both trial periods, and consisted of two standard meals served 12 hours before and 5 hours and 9 hours after dosing. No additional food intake was allowed throughout the study period. Liquid consumption was allowed ad libitum from 2 hours after dosing. Subjects were reimbursed for their time and transportation expenses, irrespective of whether they completed the study or not, in accordance with standard operating procedures implemented at our Clinical Pharmacology Centre. ${ }^{23}$ The study was performed according to the rules of Good Clinical Practice ${ }^{24}$ and the Declaration of Helsinki; ${ }^{25}$ the study protocol was approved by the Ethics Committee of Pisa University Hospital.

\section{Study design and drug administration}

This was a single-dose, randomized, two-treatment, crossover, single-blind PK/PD evaluation designed to compare generic $15 \mathrm{mg}$ tablet Meloxicam Hexal (test product) with branded Mobic $15 \mathrm{mg}$ tablets (reference product). The enrolled volunteers were randomized into two groups of twelve subjects each, and each group received the two drug treatments at two different times, with an intervening 2-week washout period. Meloxicam tablets were administered with $250 \mathrm{~mL}$ of water at 8 am after overnight fasting. Venous blood samples of $5 \mathrm{~mL}$ were collected, through an indwelling cannula placed on the forearm, into Vacutainer ${ }^{\mathrm{TM}}$ (Becton, Dickinson and Company, Franklin Lakes, NJ, USA) tubes (containing sodium heparin) at preset time intervals of 0 (predose), 0.5, 1, 2, 4, 6, 8, 12, and 24 hours after dosing. Blood samples were centrifuged at $900 \mathrm{~g}$ for 15 minutes; plasma samples were transferred into Vacutainer ${ }^{\mathrm{TM}}$ tubes (no additive) and stored at $-80^{\circ} \mathrm{C}$ until subsequent analysis.

\section{Analytical method}

Measurement of meloxicam concentrations in plasma samples was performed by validated high-performance liquid chromatography (HPLC) with ultraviolet detection, ${ }^{26}$ with some modifications. Briefly, for each sample, $200 \mu \mathrm{L}$ of plasma was added with $20 \mu \mathrm{L}$ of piroxicam $10 \mu \mathrm{g} / \mathrm{mL}$ (internal standard) plus $200 \mu \mathrm{L}$ of acetonitrile acidified with $\mathrm{H}_{3} \mathrm{PO}_{4} 0.1 \%(\mathrm{v} / \mathrm{v})$, vortexed for 20 seconds, and centrifuged for 5 minutes at $11,000 \mathrm{rpm}$ in a Heraeus Biofuge 15 (Thermo Fisher Scientific, Waltham, MA, USA) centrifuge. The clear supernatants (approximately $150 \mu \mathrm{L}$ ) were collected in vials and transferred into the autosampler. The Waters Breeze HPLC system (Waters, Milford, MA,
USA), equipped with a Waters 2476 dual-wavelength ultraviolet detector set at $355 \mathrm{~nm}$, was used. The mobile phase, consisting of potassium phosphate buffer $\left(\mathrm{K}_{2} \mathrm{HPO}_{4}\right) 20 \mathrm{mM}$ (pH 3.5) and acetonitrile (70:30, vol/vol), was isocratically pumped at a flow rate of $1.2 \mathrm{~mL} /$ minute through an Alltima $\mathrm{CN}$ chromatographic column, $150 \times 4.6 \mathrm{~mm}, 5 \mu \mathrm{m}$ (Alltech Italia, Milan, Italy). Plasma from untreated healthy subjects was used to reconstitute calibration and quality standards. In particular, for calibration standards, meloxicam was added to plasma to obtain final concentrations of 50, 10, 5, 2.5, 1.25, $0.625,0.313,0.156,0.078$, and $0.039 \mathrm{mg} / \mathrm{L}$ in a final volume of $200 \mu \mathrm{L}$. Quality controls were prepared independently from calibration-standard samples at drug concentrations of 5, 0.625, and $0.078 \mathrm{mg} / \mathrm{L}$. Samples were prepared and extracted as described above. Calibration curves of standard meloxicam were generated by plotting analyte peak areas versus drug concentrations, and linearity was estimated on at least three calibration curves obtained on 3 consecutive days. Calibration standards were also used to evaluate the parameters listed below.

\section{Determination of meloxicam plasma concentration}

Under the aforementioned chromatographic conditions, meloxicam and piroxicam had retention times of 5.93 minutes \pm 0.03 minutes and 4.53 minutes \pm 0.02 minutes, and their recovery accounted for $91 \%$ and $85 \%$, respectively. The background noise (defined as the mean amplitude of baseline oscillations) was $5 \mu \mathrm{V}$, and the limit of detection the concentration of analytes generating a signal three times higher than baseline noise - was estimated to be $0.005 \mathrm{mg} / \mathrm{L}$ on the basis of standard calibration curves. For meloxicam, the limit of quantification, defined as a sample concentration for which the method was still in the linear range, was equal to $0.039 \mathrm{mg} / \mathrm{L}$, which represented the lowest concentration among the calibration standards. The linearity of calibration curves ranged between $0.039 \mathrm{mg} / \mathrm{L}$ and $5 \mathrm{mg} / \mathrm{L}$, with a mean value of correlation coefficient for meloxicam equal to $0.989 \pm 0.003$. The linear range was calculated by means of linear regression analysis, obtaining a standard deviation of the residuals that accounted for $0.11 \mathrm{mg} / \mathrm{L} \pm 0.09 \mathrm{mg} / \mathrm{L}$. Precision and accuracy values were calculated as reported in a previous study.$^{27}$ In particular, accuracy values accounted for $88.03 \%-114.66 \%$ over the whole range of the calibration standard. Precision values ranged from $2.01 \%$ up to $7.00 \%$ over the entire range, with the exception of the lowest calibration standard $(0.039 \mathrm{mg} / \mathrm{L})$, the precision of which accounted for $17.90 \%$. For quality controls, accuracy and 
precision values were in the ranges of $87.00 \%-102.64 \%$ and $2.98 \%-13.38 \%$, respectively.

\section{Pharmacokinetic evaluations}

The actual times of sample collection were used for PK analysis of branded and generic meloxicam formulations. Area under the curve (AUC) from time 0 to 24 hours $\left(\mathrm{AUC}_{0-24}\right)$ and AUC from time 0 to 5 hours $\left(\mathrm{AUC}_{0-5}\right)$ were estimated by the linear trapezoidal method, while AUC from time 0 to infinity $\left(\mathrm{AUC}_{0-\text { inf }}\right)$ was obtained by adding to the $\mathrm{AUC}_{0-24}$ value the $\mathrm{C}_{24} / \mathrm{k}_{\mathrm{el}}$ ratio value, where $\mathrm{C}_{24}$ and $\mathrm{k}_{\mathrm{el}}$ represent the plasma concentration of meloxicam at 24 hours postdose and the elimination constant, respectively. Maximum concentration $\left(\mathrm{C}_{\max }\right)$ and the time to achieve $\mathrm{C}_{\max }\left(\mathrm{T}_{\max }\right)$ were obtained from direct visual inspection of plasma concentration versus time curves.

PK parameters were calculated by a noncompartmental method using Stata version 10.0 (StataCorp, College Station, TX, USA). Bioequivalence comparison was carried out by the statistical software SAS version 8.2 (SAS Institute, Cary, NC, USA).

\section{Pharmacodynamic evaluation}

Twenty-two hours before the first day of each experimental session, a small skin area (designed as Z1) on the anterior glabrous region of the forearm was superficially frozen. For this purpose, the tip $\left(1.8 \mathrm{~cm}^{2}\right)$ of a cylindrical copper bar, previously cooled to $-28^{\circ} \mathrm{C}$, was applied to induce a first-degree burn injury. Interindividual variability in the intensity of the hyperemic response was low and blisters were never observed, in agreement with previous results from Chassaing et al. ${ }^{13}$ Subsequently, sensory testing (ie, the assessment of baseline values of the first session) was performed 22 hours after the induction of cold injury. In the present study, mechanical hyperalgesia, as defined in the Textbook of Pain, ${ }^{28}$ was assessed by punctate-evoked pain with a von Frey device, as previously validated by Chassaing et al. ${ }^{13}$ Briefly, the quantification of pain, both at baseline and after treatment with meloxicam, was obtained by application of punctate stimuli with a constant slope of increasing punctate pressure up to the subjective perception of hyperalgesia. Mechanical pain threshold (MPT) was defined as the lowest pressure that produced a sensation of pain. Each MPT value was averaged from five separate consecutive measurements at different points within the test areas. A normal skin area (designed as Z0) on the contralateral arm was used as control. Both basal and postdosing tests were performed by the same investigator, who was blind to drug allocation. The punctate stimulation was started well outside the hyperalgesic area, where no sensation of pain was experienced, and continued centripetally in $5 \mathrm{~mm}$ steps towards the site of freeze injury until the volunteer reported a subjective perception of hyperalgesia.

\section{Statistical analysis}

Randomization was performed using a random-number table. The groups were designated as RT (subjects who received first the reference and then the test product) and TR (subjects who received first the test and then the reference product). Analysis of variance (ANOVA) for a standard $2 \times 2$ crossover design was used to evaluate period, sequence, and formulation effect. Statistical analysis was performed by parametric mixed-model accounting for subjects included in nested sequences as random effect. Log-natural transformed $\mathrm{AUC}_{0-24}$ and $\mathrm{C}_{\max }$ were used to evaluate the ratio and $90 \%$ confidence interval (CI) of the test drug over the reference product, according to Schuirmann's procedure. ${ }^{29}$ Furthermore, $\mathrm{T}_{\max }$ difference between the two meloxicam formulations was assessed by Mann-Whitney $U$ test. Finally, demographic characteristics differences between the RT and TR groups were tested by one-way ANOVA or Mann-Whitney $U$ test, according to the distribution of the variables of interest. PD subanalysis was performed by ANOVA for a standard $2 \times 2$ crossover design.

\section{Tolerability assessment}

Subjects were under continuous medical supervision at the study site throughout the study period. A physician, who was blind to the study treatment, was present throughout the study period to monitor the subjects and record possible adverse events (AEs). Every 2 hours, subjects were asked about and encouraged to report any unusual symptoms. Tolerability was assessed by investigators based on subject interviews, spontaneous reporting, vital signs, physical examination, and clinical laboratory tests (urinalysis, hematology, and blood chemistry) before and throughout the study period. Resting blood pressure, heart rate, and body temperature were monitored by investigators before drug administration (baseline) and every 2 hours throughout the study. AEs were considered serious if they were life-threatening or led to death, disability, inpatient hospitalization, or medical intervention to prevent permanent impairment or damage. All AEs were recorded on case-report forms by an investigator. Any serious AE suspected to be drug-related by investigators was reported to the ethics committee and to the local pharmacovigilance authority in accordance with the study protocol. 
Table I Summary of baseline demographic characteristics in the study population of healthy male volunteers

\begin{tabular}{|c|c|c|c|c|}
\hline $\begin{array}{l}\text { Demographic } \\
\text { characteristics }\end{array}$ & $\begin{array}{l}\text { RT group } \\
(n=I 2)\end{array}$ & $\begin{array}{l}\text { TR group } \\
(\mathrm{n}=12)\end{array}$ & $\begin{array}{l}\text { Overall study } \\
\text { population } \\
(n=24)\end{array}$ & P* \\
\hline Age, years & 27.1 (3.8) & $25.5(4.5)$ & $26.3(4.1)$ & 0.415 \\
\hline Height, m & $1.76(0.07)$ & $1.81(0.10)$ & I.79 (0.08) & 0.187 \\
\hline Weight, kg & $74.6(7.8)$ & 75.9 (II.I) & $75.2(9.3)$ & 0.738 \\
\hline $\mathrm{BMI}, \mathrm{kg} / \mathrm{m}^{2}$ & 24.0 (1.9) & 23.1 (1.9) & $23.6(1.9)$ & 0.260 \\
\hline SBP, mmHg & I $28.4(8.3)$ & $128.8(9.1)$ & $128.6(8.5)$ & 0.926 \\
\hline $\mathrm{DBP}, \mathrm{mmHg}$ & $73.3(6.0)$ & $77.0(7.2)$ & $75.2(6.7)$ & 0.188 \\
\hline $\begin{array}{l}\text { Heart rate, } \\
\text { beats/minute }\end{array}$ & 66.9 (12.7) & $76.5(12.8)$ & 71.7 (I3.4) & 0.080 \\
\hline
\end{tabular}

Notes: Values are given as mean (standard deviation). *RT versus TR. Subjects in the RT group received first the reference formulation (Mobic) and then the test formulation (Meloxicam Hexal). Subjects in the TR group received first the test formulation (Meloxicam Hexal) and then the reference formulation (Mobic).

Abbreviations: BMI, body mass index; SBP, systolic blood pressure; DBP, diastolic blood pressure.

\section{Results}

\section{Subject characteristics}

The baseline demographic characteristics of the two healthy volunteer groups are reported in Table 1. No differences between the RT and TR groups in terms of age, height, weight and BMI, systolic and diastolic blood pressure, or heart rate were found, indicating the substantial homogeneity of the two study groups.

\section{Pharmacokinetic evaluations}

Mean plasma profiles of meloxicam in the study subjects, exposed to branded (Mobic) or generic (Meloxicam Hexal) formulations, are shown in Figure 1. The respective values of estimated PK parameters are reported in Tables 2 and 3 .

\section{Pharmacokinetic bioequivalence assessment}

All the enrolled volunteers completed the study and were included in the final study population in order to perform statistical analysis. A significant period effect was found for $\mathrm{AUC}_{0-24}(P=0.03)$ but not $\mathrm{C}_{\max }(P=0.09)$, with no sequence effect for either $\mathrm{AUC}_{0-24}$ or $\mathrm{C}_{\max }(P=0.7113$ and 0.8560 , respectively), indicating a lack of formulation effect. Moreover, coefficient of variation values, estimated by ANOVA, were slightly above the recommended upper limit of $30 \%$ (34\% for $\mathrm{AUC}_{0-24}$ and $33 \%$ for $\mathrm{C}_{\max }$ ).

When comparing the generic formulation of meloxicam with the respective branded product, the test/reference ratios and 90\% CIs were $0.99(0.84-1.16)$ for $\mathrm{AUC}_{0-24}$, $0.98(0.81-1.19)$ for $\mathrm{AUC}_{0 \text {-inf }}$, and $1.05(0.89-1.23)$ for $\mathrm{C}_{\max }$. Moreover, no significant difference between the two meloxicam formulations was found with regard to $\mathrm{T}_{\max }(P=0.472)$.

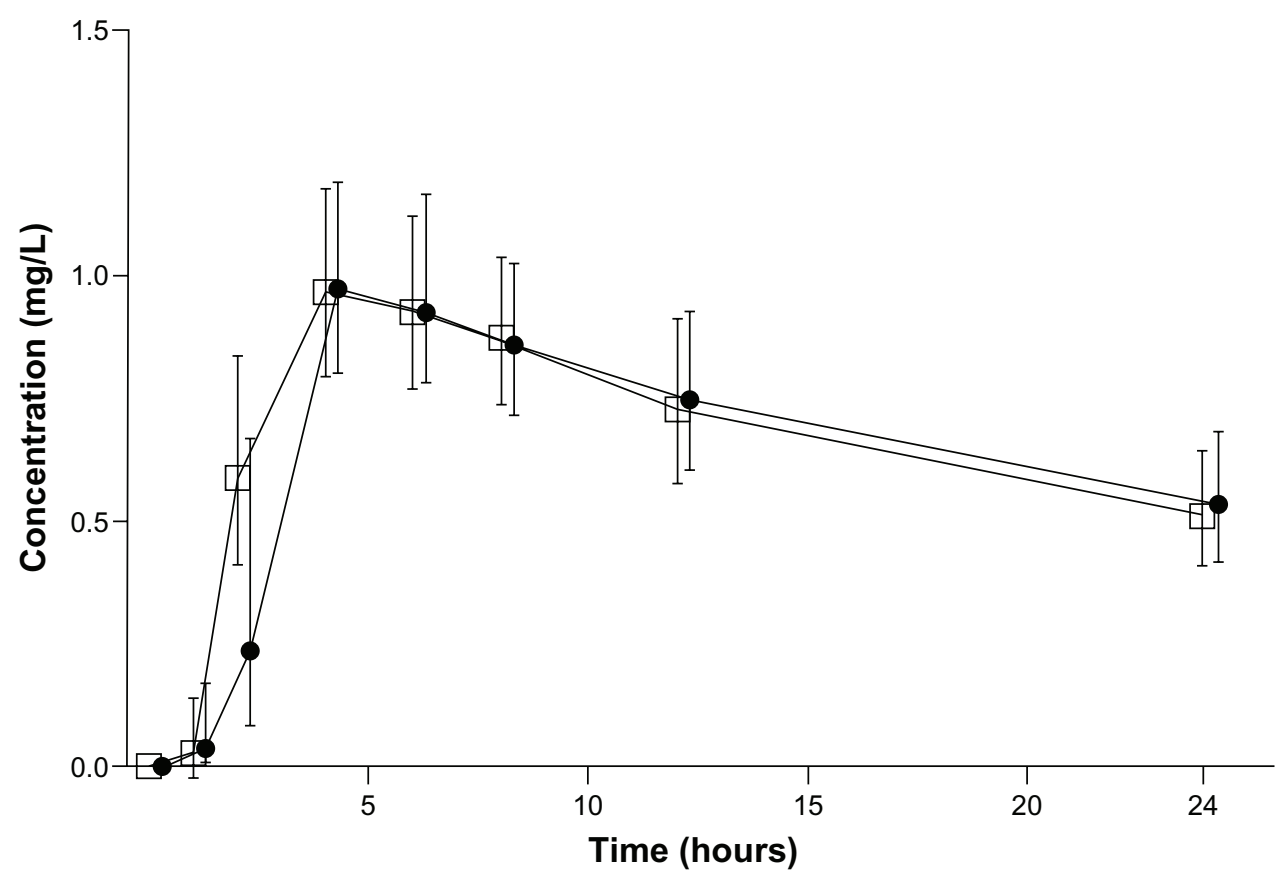

Figure I Mean concentration-time profiles in plasma following the oral administration of I5 mg tablets of branded Mobic (•) or generic Meloxicam Hexal ( $\square$ ) formulation to healthy volunteers. Each point represents the geometric mean $(95 \%$ confidence interval in vertical lines). 
Table 2 Values of plasma pharmacokinetic parameters obtained from the study population of healthy male volunteers $(\mathrm{n}=24)$ following the administration of generic (Meloxicam Hexal) and branded (Mobic) meloxicam formulations

\begin{tabular}{|c|c|c|c|c|}
\hline & Meloxicam Hexal & & Mobic & \\
\hline $\mathrm{AUC}_{(0-5)}(\mathrm{mg} \times \mathrm{h} / \mathrm{L})^{*}$ & $4.73 \pm 0.42$ & $4.29(3.56-5.17)$ & $4.29 \pm 0.34$ & $3.93(3.31-4.68)$ \\
\hline $\mathrm{AUC}_{(0-24)}(\mathrm{mg} \times \mathrm{h} / \mathrm{L})^{*}$ & $18.52 \pm 1.65$ & $16.97(14.12-20.40)$ & $18.49 \pm 1.38$ & $17.22(14.55-20.39)$ \\
\hline $\operatorname{AUC}_{(0-\text { inf })}(\mathrm{mg} \times \mathrm{h} / \mathrm{L})^{*}$ & $31.44 \pm 4.47$ & $28.11(22.06-35.81)$ & $33.25 \pm 4.03$ & $27.85(21.85-35.48)$ \\
\hline $\mathrm{C}_{\max }(\mathrm{mg} / \mathrm{L})^{*}$ & $1.32 \pm 0.01$ & $1.25(1.07-1.45)$ & $1.25 \pm 0.09$ & $1.18(1.01-1.38)$ \\
\hline $\mathrm{T}_{\text {max }}(h)^{\dagger}$ & $5.21 \pm 0.59$ & $4.00(1.00-12.00)$ & $5.75 \pm 0.53$ & $5.00(4.00-12.00)$ \\
\hline$t_{1 / 2}^{\dagger}$ & $30.61 \pm 5.35$ & I $7.83(5.23-90.04)$ & $38.05 \pm 9.90$ & $22.68(6.12-233.5 I)$ \\
\hline
\end{tabular}

Notes: *Data are reported as both arithmetic mean \pm standard error, and geometric mean with $95 \%$ confidence interval; †data are reported as both arithmetic mean \pm standard error and median with 2.5 and 97.5 percentiles.

Abbreviations: $A \cup C$, area under the time-concentration curve; $C_{\max }$, maximum plasma concentration; $T_{\max }$, time to $C_{\max } ; t_{1 / 2}$, half-life; $h$, hours;

Thus, on the basis of EMA guidelines, the $90 \%$ CI for the relevant $\mathrm{PK}$ parameters fell within the acceptability range of $0.80-1.25$, indicating pharmacokinetic bioequivalence between the two meloxicam products. Bioequivalence was not confirmed when $\mathrm{AUC}_{0-5}$ test/reference ratio with CI was calculated: the ratio was 1.09 and CI $0.92-1.30$ (Table 3).

\section{Pharmacodynamic evaluation}

A significant difference of MPT values between control (Z0) and hyperalgesic skin areas (Z1) was observed (74.8 \pm 8.7 versus $58.2 \pm 7.4, P<0.001)$. The MPT values for each area $(\mathrm{Z0}, \mathrm{Z1})$ at 5 hours are displayed in Figure 2. The mean MPT values presented a period effect, with a reduction of both Z0 and Z1 MPT in the second period of the study. No significant differences between the two formulations of meloxicam were found: $63.7 \% \pm 22.5 \%$ versus $52.3 \% \pm 13.0 \%$ at baseline $(P=0.940)$ and $88.2 \% \pm 34.5 \%$ versus $57.8 \% \pm 17.5 \%$ at 5 hours $(P=0.797)$ for Mobic and Meloxicam Hexal, respectively.

\section{Tolerability}

Two not-serious AEs were recorded during the study. A skin rash with headache developed in a volunteer 30 minutes after Mobic administration, with a spontaneous recovery after 2 hours; this $\mathrm{AE}$ was considered as probably related to the investigational medicinal product administration. One subject reported cervical pain, which was considered as drug-unrelated.

Table 3 Values of the ratios of $\mathrm{AUC}_{0-5}, \mathrm{AUC}_{0-24}, \mathrm{AUC}_{0-\text { inf }}$, and $C_{\max }$ with $90 \%$ confidence intervals

\begin{tabular}{ll}
\hline & Meloxicam Hexal/Mobic \\
\hline $\mathrm{AUC}_{0-5}(\mathrm{mg} \times \mathrm{h} / \mathrm{L})$ & $1.09(0.92-\mathrm{I} .30)$ \\
$\mathrm{AUC}_{0-24}(\mathrm{mg} \times \mathrm{h} / \mathrm{L})$ & $0.99(0.84-\mathrm{I} . \mathrm{l} 6)$ \\
$\mathrm{AUC}_{0-\text { inf }}(\mathrm{mg} \times \mathrm{h} / \mathrm{L})$ & $0.98(0.8 \mathrm{I}-\mathrm{I} .19)$ \\
$\mathrm{C}_{\max }(\mathrm{mg} / \mathrm{L})$ & $1.05(0.89-\mathrm{I}) 23)$ \\
\hline
\end{tabular}

Abbreviations: $A \cup C$, area under the time-concentration curve; $C_{\text {max }}$ maximum plasma concentration; h, hours;

\section{Discussion}

Concerns are being raised about the possibility that the quality of some marketed generic drugs may not fully reflect the results obtained in preregistration bioequivalence investigations. ${ }^{30-32}$ Substitution of branded products with generic drugs is a matter of discussion, and it is often regarded with skepticism by both health-care providers and patients. Current bioequivalence requirements are based on a measure of average bioequivalence; however, there are concerns that the use of such a measure might be inappropriate in the case of drugs with high intrasubject or intersubject variability, like analgesic medications. ${ }^{33}$ Moreover, in some countries, the lack of open access to documentation of preregistration studies represents a point of weakness of the generic drug market. Indeed, prescribing physicians cannot access any information on the preregistration development of generic drugs, and they can only trust that the regulatory authority approved a specific generic formulation in full accordance with recommended procedures. If a generic drug was

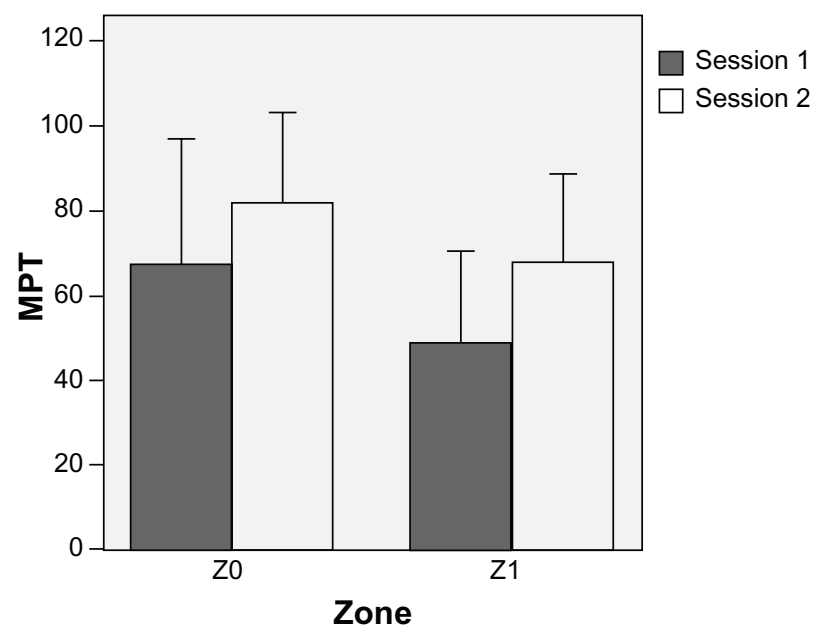

Figure 2 Mechanical pain threshold (MPT) at 5 hours determined by a von Frey device over the two sessions on control (ZO) and hyperalgesic (ZI) skin zone. Each session was performed according to the crossover study design (period I and period 2). Data are expressed as means \pm standard deviation (vertical lines). 
approved with only the minimum of regulatory requirements for its registration (ie, by an in vitro study), the postmarketing interchangeability of that generic drug with its respective branded product might not be warranted. Based on these considerations, the present study was performed: (1) to compare the PK profiles of two meloxicam formulations (branded and generic), containing different excipient ingredients, in order to verify whether the generic formulation conforms with its respective branded product, in accordance with the criteria established by EMA guidelines and Italian laws; and (2) to evaluate whether a PD end point comparison can be related to PK analysis and is suitable for improving the control of quality pursued by postmarketing studies.

In accordance with EMA guidelines, estimated PK parameters $\mathrm{AUC}_{0-24}$ and $\mathrm{C}_{\max }$ fell within the acceptability range of $0.80-1.25$, indicating the interchangeability between the two meloxicam products and confirming that $\mathrm{PK}$ analysis is consistent with $\mathrm{PD}$ comparison results. Bioequivalence was not confirmed in a subanalysis of $\mathrm{AUC}_{0-5}$ due to the huge variation of standard deviation in the first phases of PK (dissolution/absorption), but PD testing at 5 hours showed that there was no difference between formulations in terms of drug effects.

It is worth noting that the $\mathrm{AUC}_{0-5} \mathrm{PK}$ end point is not considered by current guidelines, but the present study focused attention on this end point to investigate whether it could predict the analgesic effect early after drug administration. Moreover, the PD test was considered an explorative analysis, and taking into account the huge variation obtained in this analysis, the PD results led to a more probable beta error, thus making arguable the acceptance of the null hypothesis (drug equivalence). Since large intersubject variability of $\mathrm{AUC}_{0-5}$ values and $P D$ results hampered our analysis, this issue should be carefully considered in future studies, where the collection of plasma samples at earlier time points would allow more reliability and confidence in the results to be obtained.

The time-point collection (24 hours), which was not long enough to cover two drug half-lives, could appear as a limitation of the present study. However, the main study objective was not to investigate the complete PK profiles of meloxicam in healthy volunteers, since this information has been previously provided by other authors, ${ }^{19-22,34,35}$ but rather to compare PK and PD patterns between branded and generic meloxicam. On this basis, we performed a blood-sampling collection during the dissolution, absorption, and distribution PK phases, assuming that the elimination phase would not be significantly affected by the pharmaceutical formulation. Consistently with this assumption, when the present PK results were compared with those published earlier, ${ }^{36}$ the length of time sampling appeared to be long enough to allow a good estimation of meloxicam PK.

The present results are in agreement with previous PK data ${ }^{19-22,34}$ obtained on branded meloxicam in healthy volunteers. When considering the study performed by MarcelínJiménez et $\mathrm{al}^{35}$ in a Mexican female population, although the $T_{\max }$ value was similar to that estimated in the present study, both $\mathrm{C}_{\max }$ and $\mathrm{AUC}_{0-24}$ values were found to be higher. However, differences in sex, body weight, genetic profile, and pharmaceutical composition of tablets are likely to explain the differences between the Italian and Mexican studies.

If two drugs are proven to be interchangeable, highquality manufacturing standards are followed, and stringent quality controls are ensured over time, then the switch from a brand formulation to a generic one should be acceptable in most cases, without any expected noticeable variation in efficacy or tolerability. ${ }^{37}$ However, postmarketing evaluations have highlighted cases of lack of equivalence between generic and branded drugs. One case is the study by Del Tacca et al, ${ }^{2}$ who compared brand amoxicillin with two generic formulations available in the Italian market, and did not confirm interchangeability for one of the two generic products. In another study, the lack of postmarketing therapeutic equivalence between branded and generic cefuroxime was found to significantly influence the incidence of postoperative infections in adult patients undergoing coronary artery bypass grafting surgery. ${ }^{6}$ Development of breakthrough seizures and increased seizure frequency was observed after switching to marketed generic antiepileptic drugs, possibly due to changes in serum concentrations. ${ }^{4}$ Likewise, psychiatric symptom relapse in a stabilized patient, resulting in hospitalization, after a switch to a marketed generic formulation of clozapine, ${ }^{38}$ has been reported. In the postmarketing setting, plasma levels of venlafaxine and its active metabolite O-desmethylvenlafaxine were found to be significantly higher in subjects given a generic product, as compared with those treated with the branded formulation. ${ }^{11}$

Overall, bioequivalence studies should be considered not only as a part of the regulatory process, which allows the marketing authorization of a generic drug, but also as valuable scientific tools suitable for evaluating the "goodness" (ie, the quality) of generic drugs available in the pharmaceutical market, thus reducing the risks associated with a lack of interchangeability persistence. In particular, by means of comparative bioequivalence studies between generic products and their respective branded drugs in the postmarketing setting, we could assure for both patients and 
prescribing physicians not only a pharmaceutical but also a pharmacological (ie, PK and/or PD) equivalence, which would add support to the expected clinical efficacy of the marketed generic drug. Accordingly, an efficient program of postmarketing drug evaluation should take into account $\mathrm{PK}$, and in some (specific) cases PD studies with single or multiple doses (when reaching a steady-state level is deemed relevant). In this respect, the present study suggests that $\mathrm{PD}$ evaluations may represent simple and useful tools, which might improve the quality of postmarketing comparisons. Indeed, when the PD equivalence analysis of generic and branded formulations completes the PK evaluation, the existence of actual interchangeability can be better documented.

\section{Conclusion}

The results of the present postmarketing study demonstrate that the PK profiles of the branded and generic meloxicam formulations confirm the regulatory criteria for bioequivalence. Taken together with the PD data, these results indicate that both formulations can be used interchangeably in clinical practice.

\section{Acknowledgments}

The authors wish to acknowledge Dr Catia Castiglioni for her invaluable support to the clinical management of study subjects, as well as Dr Ciro Conversano for his careful psychological evaluation of healthy volunteers before their enrollment.

\section{Disclosure}

This study was supported by unconditional funds provided by Boehringer Ingelheim. The authors report no other conflicts of interest in this work.

\section{References}

1. European Medicines Agency. Guideline on the investigation of bioequivalence. 2010. Available from: http://www.emea.europa.eu/docs/ en_GB/document_library/Scientific_guideline/2010/01/WC500070039. pdf. Accessed July 24, 2012.

2. Del Tacca M, Pasqualetti G, Di Paolo A, et al. Lack of pharmacokinetic bioequivalence between generic and branded amoxicillin formulations. A post-marketing clinical study on healthy volunteers. $\mathrm{Br} J$ Clin Pharmacol. 2009;68:34-42.

3. Crawford P, Feely M, Guberman A, Kramer G. Are there potential problems with generic substitution of antiepileptic drugs? A review of issues. Seizure. 2006;15:165-176.

4. Berg MJ, Gross RA, Tomaszewski KJ, Zingaro WM, Haskins LS. Generic substitution in the treatment of epilepsy: case evidence of breakthrough seizures. Neurology. 2008;71:525-530.

5. LeLorier J, Duh MS, Paradis PE, et al. Clinical consequences of generic substitution of lamotrigine for patients with epilepsy. Neurology. 2008;70:2179-2186.
6. Mastoraki E, Michalopoulos A, Kriaras I, et al. Incidence of postoperative infections in patients undergoing coronary artery bypass grafting surgery receiving antimicrobial prophylaxis with original and generic cefuroxime. $J$ Infect. 2008;56:35-39.

7. Halkin H, Shapiro J, Kurnik D, Loebstein R, Shalev V, Kokia E. Increased warfarin doses and decreased international normalized ratio response after nationwide generic switching. Clin Pharmacol Ther. 2003;74: 215-221.

8. Elkoshi Z, Behr D, Mirimsky A, Tsvetkov I, Danon A. Multiple-dose studies can be a more sensitive assessment for bioequivalence than single-dose studies: the case with omeprazole. Clin Drug Invest. 2002;22:585-592.

9. Shimatani T, Inoue M, Kuroiwa T, Xu J, Mieno H, Tazuma S. Acidsuppressive effects of generic omeprazole: comparison of three brands of generic omeprazole with original omeprazole. Dig Liver Dis. 2006; 38:554-559.

10. Reiffel JA. Issues in the use of generic antiarrhythmic drugs. Curr Opin Cardiol. 2001;16:23-29.

11. Chenu F, Batten LA, Zernig G, Ladstaetter E, Hébert C, Blier P. Comparison of pharmacokinetic profiles of brand-name and generic formulations of citalopram and venlafaxine: a crossover study. J Clin Psychiatry. 2009;70:958-966.

12. Meredith PA. Potential concerns about generic substitution: bioequivalence versus therapeutic equivalence of different amlodipine salt forms. Curr Med Res Opin. 2009;25:2179-2189.

13. Chassaing C, Schmidt J, Eschalier A, Cardot JM, Dubray C. Hyperalgesia induced by cutaneous freeze injury for testing analgesics in healthy volunteers. Br J Clin Pharmacol. 2006;4:389-397.

14. Meineke I, Türck D. Population pharmacokinetic analysis of meloxicam in rheumatoid arthritis patients. Br J Clin Pharmacol. 2003;55:32-38.

15. Stening K, Eriksson O, Wahren L, Berg G, Hammar M, Blomqvist A. Pain sensations to the cold pressor test in normally menstruating women: comparison with men and relation to menstrual phase and serum sex steroid levels. Am J Physiol Regul Integr Comp Physiol. 2007;293: R1711-R1716.

16. Ring C, Veldhuijzen van Zanten JJ, Kavussanu M. Effects of sex, phase of the menstrual cycle and gonadal hormones on pain in healthy humans. Biol Psychol. 2009;81:189-191.

17. Kowalczyk WJ, Sullivan MA, Evans SM, Bisaga AM, Vosburg SK, Comer SD. Sex differences and hormonal influences on response to mechanical pressure pain in humans. J Pain. 2010;11:330-342.

18. Teepker M, Peters M, Vedder H, Schepelmann K, Lautenbacher S. Menstrual variation in experimental pain: correlation with gonadal hormones. Neuropsychobiology. 2010;61:131-140.

19. Gschwend MH, Erenmemişoğlu A, Martin W, Tamur U, Kanzik I, Hincal AA. Pharmacokinetic and bioequivalence study of meloxicam tablets in healthy male subjects. Arzneimittelforschung. 2007;57: 264-268.

20. Tangsucharit P, Kampan J, Kanjanawart S, et al. Bioequivalence study of two meloxicam tablet formulations after single-dose administration in healthy Thai male volunteers. Int J Clin Pharmacol Ther. 2009;47:638-642.

21. Hasan SM, Shoaib MH, Hassan F, Rehman IU. Bioequivalence studies of two brands of meloxicam tablets in healthy Pakistani volunteers. Pak J Pharm Sci. 2009;22:199-204.

22. Ahmad M, Murtaza G, Akhtar N, et al. Bioequivalence study of two brands of meloxicam tablets in healthy human Pakistani male subjects. Acta Pol Pharm. 2011;68:115-119.

23. Pasqualetti G, Gori G, Blandizzi C, Del Tacca M. Healthy volunteers and early phases of clinical experimentation. Eur J Clin Pharmacol. 2010;66:647-653.

24. European Medicines Agency. Guideline for good clinical practice. 2002. Available from: http://www.ema.europa.eu/docs/en_GB/ document_library/Scientific_guideline/2009/09/WC500002874.pdf. Accessed July 24, 2012. 
25. World Medical Association Declaration of Helsinki 2008. Ethical principles for medical research involving human subjects. 2008. Available from: http://www.wma.net/en/30publications/10policies/b3/17c.pdf. Accessed July 24, 2012.

26. Bae JW, Kim MJ, Jang CG, Lee SY. Determination of meloxicam in human plasma using a HPLC method with UV detection and its application to a pharmacokinetic study. J Chromatogr B Analyt Technol Biomed Life Sci. 2007;859:69-73.

27. Polillo M, Tascini C, Lastella $M$, et al. A rapid high-performance liquid chromatography method to measure linezolid and daptomycin concentrations in human plasma. Ther Drug Monit. 2010;32: 200-205.

28. Meyer RA, Campbell JN, Raja SN. Peripheral neural mechanisms of nociception. In: Wall PD, Melzack R, Bonica JJ, editors. Textbook of Pain. New York: Churchill Livingstone; 1995:13-44.

29. Schuirmann DJ. A comparison of the two one-sided tests procedure and the power approach for assessing the equivalence of average bioavailability. J Pharmacokinet Biopharm. 1987;15:657-680.

30. Chen ML, Shah V, Patnaik R, et al. Bioavailability and bioequivalence: an FDA regulatory overview. Pharm Res. 2001;18:1645-1650.

31. Yu LX, Amidon GL, Polli JE, et al. Biopharmaceutics classification system: the scientific basis for biowaiver extensions. Pharm Res. 2002;19:921-925.
32. World Health Organization. Proposal to waive in vivo bioequivalence requirements for the WHO model list of essential medicines: immediate release, solid oral dosage forms. 2005. Available from: http:// www.who.int/medicines/services/expertcommittees/pharmprep/ QAS04_109Rev1_Waive_invivo_bioequiv.pdf. Accessed July 24, 2012.

33. Meredith P. Bioequivalence and other unresolved issues in generic drug substitution. Clin Ther. 2003;25:2875-2890.

34. Türck D, Roth W, Busch U. A review of the clinical pharmacokinetics of meloxicam. Br J Rheumatol. 1996;35 Suppl 1:13-16.

35. Marcelín-Jiménez G, Hernández JA, Angeles AP, et al. Bioequivalence evaluation of two brands of meloxicam tablets (Promotion and Mobicox): pharmacokinetics in a healthy female Mexican population. Biopharm Drug Dispos. 2005;26:167-171.

36. Davies NM, Skjodt NM. Clinical pharmacokinetics of meloxicam. A cyclo-oxygenase-2 preferential nonsteroidal anti-inflammatory drug. Clin Pharmacokinet. 1999;36:115-126.

37. Perry R. Perspectives on the bioequivalence and therapeutic equivalence of generic formulations: an overview of the landscape. Clin Ther. 2010;32:1796-1797.

38. Alvarez CA, Mascarenas C, Timmerman I. Increasing psychosis in a patient switched from Clozaril to generic clozapine. Am J Psychiatry. 2006;163(4):746.
Therapeutics and Clinical Risk Management

\section{Publish your work in this journal}

Therapeutics and Clinical Risk Management is an international, peerreviewed journal of clinical therapeutics and risk management, focusing on concise rapid reporting of clinical studies in all therapeutic areas, outcomes, safety, and programs for the effective, safe, and sustained use of medicines. This journal is indexed on PubMed Central, CAS,

\section{Dovepress}

EMBase, Scopus and the Elsevier Bibliographic databases. The manuscript management system is completely online and includes a very quick and fair peer-review system, which is all easy to use. Visit http://www.dovepress.com/testimonials.php to read real quotes from published authors.

Submit your manuscript here: http://www.dovepress.com/therapeutics-and-clinical-risk-management-journal 